PROCEEDINGS OF THE

AMERICAN MATHEMATICAL SOCIETY

Volume 137, Number 2, February 2009, Pages 505-509

S 0002-9939(08)09600-7

Article electronically published on September 15, 2008

\title{
HOMOLOGY OF REAL ALGEBRAIC VARIETIES AND MORPHISMS TO SPHERES
}

\author{
ALİ ÖZTÜRK \\ (Communicated by Ted Chinburg)
}

\begin{abstract}
Let $X$ and $Y$ be affine nonsingular real algebraic varieties. One of the classical problems in real algebraic geometry is whether a given $C^{\infty}$ mapping $f: X \rightarrow Y$ can be approximated by entire rational mappings in the space of $C^{\infty}$ mappings. In this work, we obtain some sufficient conditions in the case when $Y$ is the standard sphere $S^{n}$.
\end{abstract}

\section{INTRODUCTION AND THE RESULTS}

Given two nonsingular affine real algebraic varieties $X$ and $Y$, we regard the set $R(X, Y)$ of all entire rational maps from $X$ into $Y$ as a subset of the space $C^{\infty}(X, Y)$ of all $C^{\infty}$ maps from $X$ into $Y$ endowed with the $C^{\infty}$-topology.

In this study we focus on the question of when $C^{\infty}$ maps between nonsingular affine real algebraic varieties can be approximated by entire rational maps. If $X$ is compact and nonsingular, as indicated by the classical Stone-Weierstrass approximation theorem, every $C^{\infty}$ mapping $f: X \rightarrow \mathbb{R}^{n}$ can be approximated by the polynomial maps in $C^{\infty}\left(X, \mathbb{R}^{n}\right)$. In particular, every $C^{\infty}$ mapping from $X$ into Euclidean space can be approximated by entire rational maps in the $C^{\infty}$-topology. The general idea is to try to extend this result to different target spaces. The next natural case is to take the standard $n$-dimensional unit sphere

$$
S^{n}=\left\{x_{0}, \ldots, x_{n} \in \mathbb{R}^{n+1} \mid x_{0}^{2}+\ldots+x_{n}^{2}=1\right\}
$$

as a target space. In this case, the approximation problem becomes very difficult. There are some positive results in this direction. First, Ivanov proved that the smooth map $f: X \rightarrow S^{1}$ can be approximated by entire rational maps from $X$ to $S^{1}$ if and only if $f^{*}(u)$ belongs to $H_{\text {alg }}^{1}\left(X, \mathbb{Z}_{2}\right)$, where $u$ is a generator of $H_{\text {alg }}^{1}\left(S^{1}, \mathbb{Z}_{2}\right)$ 9. After that Bochnak and Kucharz extended this result to $S^{2}$ and obtained some partial results for $S^{4}$ 3, 5. There are also some negative results. Loday showed that any polynomial map from $T^{n}$ to $S^{n}$ is null homotopic 10. Bochnak and Kucharz proved that any entire rational map from $X \times S^{2 n-k}$ to $S^{2 n}$ is null homotopic, where $k$ is the dimension of $X$ and $k<2 n$ [3] (see also [11, 12]).

We examine this approximation problem for maps to spheres that factor through the real or the complex projective spaces. Our main results follow.

Received by the editors November 10, 2005, and, in revised form, March 2, 2008.

2000 Mathematics Subject Classification. Primary 14P25; Secondary 14P05.

Key words and phrases. Real algebraic varieties, algebraic homology, regular morphisms.

(C)2008 American Mathematical Society 
Theorem 1.1. Let $X^{2 n}$ be a nonsingular compact orientable real algebraic variety and $f: X^{2 n} \rightarrow S^{2 n}$ be a continuous map. If there is a cohomology class $u \in$ $H_{\mathbb{C}-\text { alg }}^{2}(X, \mathbb{Z})$ such that $u^{n}=f^{*}(\alpha)$, where $\alpha \in H^{2 n}\left(S^{2 n}, \mathbb{Z}\right)$ is a generator, then $f$ is homotopic to an entire rational map.

The next theorem gives a partial answer to the converse of the above theorem.

Theorem 1.2. Let $f: X^{2 n} \rightarrow S^{2 n}$ be a continuous map where $X^{2 n}$ is a nonsingular compact orientable real algebraic variety. If there is an entire rational map $\tilde{f}$ : $X \rightarrow \mathbb{C P}^{n}$ such that $\pi \circ \tilde{f}$ is homotopic to $f$, then there is a cohomology class $u \in H_{\mathbb{C}-\text { alg }}^{2}(X, \mathbb{Z})$ such that $u^{n}=f^{*}(\alpha)$, where $\alpha$ is a generator of $H^{2 n}\left(S^{2 n}, \mathbb{Z}\right)$.

For a nonorientable real algebraic variety we have the following result.

Theorem 1.3. Let $X^{n}$ be a nonorientable, closed, nonsingular variety and $f: X \rightarrow$ $S^{n}$ be a continuous map. If there is some $v \in H_{\text {alg }}^{1}\left(X, \mathbb{Z}_{2}\right)$ such that $v^{n}=f^{*}(\alpha)$ and $\alpha \in H^{n}\left(S^{n}, \mathbb{Z}_{2}\right)$ is a generator, then $f$ is homotopic to an entire rational map.

Remark 1.4. Clearly in Theorems 1.1 and 1.3 the assumption of the existence of certain algebraic cohomology classes on $X$ is not necessary since the identity map $i d: S^{k} \rightarrow S^{k}$ is entire rational for any $k$.

Example 1.5. Let $M$ be a smooth closed orientable manifold of dimension $2 n$ and $u \in H^{2}\left(M \sharp \mathbb{C P}^{n}, \mathbb{Z}\right)$ be such that $u^{n} \in H^{2 n}\left(M \sharp \mathbb{C P}^{n}, \mathbb{Z}\right)$ is a generator. Then, by Theorem 1.2 of $[6], M \sharp \mathbb{C P}^{n}$ has an algebraic model $X$ such that $u \in H_{\mathbb{C}-\text { alg }}^{2}(X, \mathbb{Z})$. Hence, there are plenty of examples of algebraic varieties satisfying the hypothesis of Theorem 1.1

Example 1.6. Let $M$ be a smooth closed orientable manifold of dimension $n$ and $w \in H^{1}\left(M, \mathbb{Z}_{2}\right)$ such that $w^{n}$ is a generator of $H^{n}\left(M, \mathbb{Z}_{2}\right)$. Let $G$ be a subgroup of $H^{1}\left(M, \mathbb{Z}_{2}\right)$ generated by $w$ and $w_{1}(M)$. Then, by Theorem 4.1 of [7, there exist an algebraic model $X$ of $M$ and a diffeomorphism $h: X \rightarrow M$ such that $h^{*}(G)=H_{\text {alg }}^{1}\left(X, \mathbb{Z}_{2}\right)$.

In general, let $N$ be any smooth manifold of dimension $n$. Then $M=N \sharp \mathbb{R} \mathbb{P}^{n}$ has a class $w \in H^{1}\left(M, \mathbb{Z}_{2}\right)$ such that $w^{n}$ is a generator of $H^{n}\left(M, \mathbb{Z}_{2}\right)$ and hence by the above paragraph there exists an algebraic model $X$ of $M$ such that $w \in H_{\text {alg }}^{1}\left(X, \mathbb{Z}_{2}\right)$ with $w^{n}$ a generator of $H^{n}\left(X, \mathbb{Z}_{2}\right)$.

\section{Proofs}

All real algebraic varieties under consideration in this report are nonsingular. It is well known that real projective varieties are affine (cf. Proposition 2.4.1 [1] or Theorem 3.4.4 [2]). Moreover, compact affine real algebraic varieties are projective (cf. Corollary 2.5.14 [1]), and therefore we do not distinguish between real compact affine varieties and real projective varieties.

For real algebraic varieties $X \subseteq \mathbb{R}^{r}$ and $Y \subseteq \mathbb{R}^{s}$, a map $F: X \rightarrow Y$ is said to be entire rational if there exist $f_{i}, g_{i} \in \mathbb{R}\left[x_{1}, \ldots, x_{r}\right], i=1, \ldots, s$, such that each $g_{i}$ vanishes nowhere on $X$ and $F=\left(f_{1} / g_{1}, \ldots, f_{s} / g_{s}\right)$. We say $X$ and $Y$ are isomorphic if there are entire rational maps $F: X \rightarrow Y$ and $G: Y \rightarrow X$ such that $F \circ G=i d_{Y}$ and $G \circ F=i d_{X}$. Isomorphic algebraic varieties will be regarded as the same. 
An algebraic homology group $H_{k}^{a l g}(X, R)\left(R=\mathbb{Z}\right.$ or $\left.\mathbb{Z}_{2}\right)$ is defined as the subgroup of $H_{k}(X, R)$ generated by the compact real algebraic subsets of $X$. Define $H_{a l g}^{*}(X, R)$ to be the Poincaré dual of the groups $H_{*}^{a l g}(X, R)$ where it is defined.

For a compact nonsingular affine real algebraic variety $X, H_{\mathbb{C}-\text { alg }}^{2 k}(X, \mathbb{Z})$, consisting of the elements which are the restriction of the classes in $H^{2 k}\left(X_{\mathbb{C}}, \mathbb{Z}\right)$ via the projective nonsingular complexification map $j: X \rightarrow X_{\mathbb{C}}$ whose Poincaré dual is represented by complex algebraic cycles is defined to be the subgroup of $H^{2 k}(X, \mathbb{Z})$ 4]. We refer the reader for the basic definitions and facts about real algebraic geometry to [1, 2].

First we have a purely topological result.

Lemma 2.1. Let $M$ be a smooth closed orientable manifold of dimension $2 n$ and $f: M \rightarrow S^{2 n}$ be any smooth map. Then there is a smooth map $\tilde{f}: M \rightarrow \mathbb{C P}^{n}$ such that the diagram

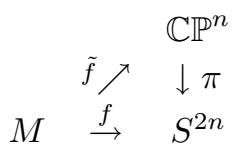

commutes up to homotopy if and only if there is a cohomology class $u \in H^{2}(M, \mathbb{Z})$ such that $u^{n}=f^{*}(\alpha)$, where $\alpha \in H^{2 n}\left(S^{2 n}, \mathbb{Z}\right)$ is a generator.

Proof of Lemma 2.1. By the Hopf classification theorem there is a continuous degree one map $\pi: \mathbb{C P}^{n} \rightarrow S^{2 n}$ (cf. Theorem 11.6, p. 300 [8]). Next, assume that such an $\tilde{f}$ exists. Then,

$$
\begin{aligned}
f^{*}(\alpha) & =(\pi \circ \tilde{f})^{*}(\alpha) \\
& =\left(\tilde{f}^{*} \circ \pi^{*}\right)(\alpha) \\
& =\tilde{f}^{*}\left(a^{n}\right) \quad(\pi \text { is a degree one map }) \\
& =\left(\tilde{f}^{*}(a)\right)^{n} \\
& =u^{n}
\end{aligned}
$$

here $a \in H^{2}\left(\mathbb{C P}^{n}, \mathbb{Z}\right)$ is a generator and $u=\tilde{f}^{*}(a)$. So, one side has been proved.

Conversely, assume that there is a cohomology class $u \in H^{2}(M, \mathbb{Z})$ in the form of $u^{n}=f^{*}(\alpha)$. Let $\tilde{f}: M \rightarrow \mathbb{C P}^{\infty}$, which is the Eilenberg-Mac Lane space $K(\mathbb{Z}, 2)$, be a map such that $\tilde{f}^{*}(a)=u$, where $a \in H^{2}\left(\mathbb{C P}^{\infty}, \mathbb{Z}\right)$ is a generator. Since $M$ is $2 n$-dimensional, we can change $\tilde{f}$ by a homotopy so that $\tilde{f}(M) \subseteq \mathbb{C P}^{n} \subseteq \mathbb{C P}^{\infty}$, where $\mathbb{C P}^{n}$ is the $2 n$-th skeleton of $\mathbb{C P}^{\infty}$. Now we can assume that $\tilde{f}: M \rightarrow \mathbb{C P}^{n}$ is a map such that $\tilde{f}^{*}(a)=u$. Then, $\left(\tilde{f}^{*}(a)\right)^{n}=u^{n}$, where $a^{n} \in H^{2 n}\left(\mathbb{C P}^{n}, \mathbb{Z}\right)$. Since $a^{n}=\pi^{*}(\alpha)$, we get

$$
\begin{aligned}
(\pi \circ \tilde{f})^{*}(\alpha) & =\tilde{f}^{*}\left(\pi^{*}(\alpha)\right) \\
& =u^{n} \\
& =f^{*}(\alpha) .
\end{aligned}
$$

Thus, $\pi \circ \tilde{f}$ and $f$ have the same degree and hence $\pi \circ \tilde{f}$ and $f$ are homotopic.

Proof of Theorem 1.1. By Lemma 2.1, there is a map $\tilde{f}: X \rightarrow \mathbb{C P}^{n}$ such that $\pi \circ \tilde{f}$ is homotopic to $f$. The pull-back complex line bundle $\tilde{f}^{*}\left(\gamma_{n, 1}\right)$, where $\left(\gamma_{n, 1}\right) \rightarrow \mathbb{C P}^{n}$ is the canonical line bundle over $\mathbb{C P}^{n}$, is strongly algebraic because its Chern class, $c_{1}\left(\tilde{f}^{*}\left(\gamma_{n, 1}\right)\right)=u$, is in $H_{\mathbb{C}-a l g}^{2}(X, \mathbb{Z})$ (cf. Remark 5.4 [4]). Now by Theorem 13.3.1 
of [2] the map $\tilde{f}$ classifying the pull-back bundle can be homotoped to an entire rational map.

Proof of Theorem 1.2. Since $\pi: \mathbb{C P}^{n} \rightarrow S^{2 n}$ has degree one we have $\pi^{*}(\alpha)=$ $a^{n}$, where $a \in H^{2}\left(\mathbb{C P}^{n}, \mathbb{Z}\right)$ is a generator. It is well known that $H^{2}\left(\mathbb{C P}^{n}, \mathbb{Z}\right)=$ $H_{\mathbb{C}-\text { alg }}^{2}\left(\mathbb{C P}^{n}, \mathbb{Z}\right)$. Now, let $u=\tilde{f}^{*}(a)$. Then, $u \in H_{\mathbb{C}-\text { alg }}^{2}(X, \mathbb{Z})$ because $\tilde{f}$ is an entire rational map. By assumption, $\pi \circ \tilde{f}$ is homotopic to $f$ and hence we get

$$
f^{*}(\alpha)=(\tilde{f})^{*} \pi^{*}(\alpha)=\tilde{f}^{*}\left(a^{n}\right)=\left(\tilde{f}^{*}(a)\right)^{*}=u^{n} .
$$

Next we give a similar proof for Theorem 1.3 using the real projective space instead of the complex projective space. Let $\pi: \mathbb{R P}^{n} \rightarrow S^{n}$ be an entire rational map defined by

$$
\pi\left(\left[x_{0}: \ldots: x_{n}\right]\right)=\|x\|^{-2}\left(2 x_{0} x_{n}, \ldots, 2 x_{n-1} x_{n},\left(\sum_{i=0}^{n-1} x_{i}^{2}\right)-x_{n}^{2}\right) .
$$

Then the following diagram commutes:

$\begin{array}{ccc}\mathbb{R P}^{n} & \stackrel{\pi}{\rightarrow} & S^{n} \\ \varphi \uparrow & & \uparrow i \\ \mathbb{R}^{n} & \stackrel{\phi^{-1}}{\rightarrow} & S^{n}-(N),\end{array}$

where $N=(0,0, \ldots, 1)$ is the north pole of $S^{n}, \phi$ is the stereographic projection, $\varphi$ is the embedding defined by $\varphi\left(x_{1}, \ldots, x_{n}\right)=\left[x_{1}: \ldots: x_{n}: 1\right]$, and $i$ is the inclusion map. We may consider $\pi$ as an extension of $\phi^{-1}$ so that $\operatorname{deg}(\pi)=1$, where we consider the $\mathbb{Z}_{2}$ degree when $n$ is even.

Lemma 2.2. Let $M^{n}$ be a nonorientable manifold and $f: M^{n} \rightarrow S^{n}$ be a continuous map. Then there is a continuous map $\tilde{f}: M^{n} \rightarrow \mathbb{R P}^{n}$ such that the diagram

$$
\begin{aligned}
& \mathbb{R} \mathbb{P}^{n} \\
& \tilde{f} \nearrow \downarrow \pi \\
& M^{n} \stackrel{f}{\rightarrow} S^{n}
\end{aligned}
$$

commutes up to homotopy if and only if there is a cohomology class $v \in H^{1}\left(M, \mathbb{Z}_{2}\right)$ such that $v^{n}=f^{*}(\alpha)$, where $\alpha \in H^{n}\left(S^{n}, \mathbb{Z}_{2}\right)$ is a generator.

Proof of Lemma 2.2. Assume that there exists an $\tilde{f}$. Then,

$$
\begin{aligned}
f^{*}(\alpha) & =(\pi \circ \tilde{f})^{*}(\alpha) \\
& =\left(\tilde{f}^{*} \circ \pi^{*}\right)(\alpha) \\
& =\tilde{f}^{*}\left(a^{n}\right) \quad(\pi \text { is a degree one map }) \\
& =\left(\tilde{f}^{*}(a)\right)^{n} \\
& =v^{n}
\end{aligned}
$$

here $a \in H^{1}\left(\mathbb{R P}^{n}, \mathbb{Z}_{2}\right)$ is a generator and $v=\tilde{f}^{*}(a)$.

Conversely, assume that $v \in H^{1}\left(M^{n}, \mathbb{Z}_{2}\right)$ such that $v^{n}=f^{*}(\alpha)$. Let $\tilde{f}: M^{n} \rightarrow$ $\mathbb{R P}^{\infty}=K\left(\mathbb{Z}_{2}, 1\right)$ be a map such that $\tilde{f}^{*}(a)=v$, where $a \in H^{1}\left(\mathbb{R P}^{\infty}, \mathbb{Z}_{2}\right)$ is a generator. Since $M^{n}$ is $n$-dimensional, we can change $\tilde{f}$ by a homotopy so that $\tilde{f}\left(M^{n}\right) \subseteq \mathbb{R P}^{n} \subseteq \mathbb{R} \mathbb{P}^{\infty}$, where $\mathbb{R P}^{n}$ is the $n$-th skeleton of $\mathbb{R} \mathbb{P}^{\infty}$. Now we assume that $\tilde{f}: M^{n} \rightarrow \mathbb{R} \mathbb{P}^{n}$ is a map such that $\tilde{f}^{*}(a)=v$, where we can assume $a \in$ 
$H^{1}\left(\mathbb{R} \mathbb{P}^{n}, \mathbb{Z}_{2}\right)$. Then, $\left(\tilde{f}^{*}(a)\right)^{n}=v^{n}$. Since $a^{n}=\pi^{*}(\alpha)$, we get

$$
\begin{aligned}
(\pi \circ \tilde{f})^{*}(\alpha) & =\tilde{f}^{*}\left(\pi^{*}(\alpha)\right) \\
& =v^{n} \\
& =f^{*}(\alpha) .
\end{aligned}
$$

Thus, we get that $\pi \circ \tilde{f}$ and $f$ have the same $\mathbb{Z}_{2}$ degree and thus they are homotopic.

Proof of Theorem 1.3, By Lemma 2.2, there is an $\tilde{f}: X \rightarrow \mathbb{R}^{n}$ such that $\pi \circ \tilde{f}$ is homotopic to $f$. The pull-back real line bundle $\tilde{f}^{*}\left(\gamma_{n, 1}\right)$, where $\left(\gamma_{n, 1}\right) \rightarrow \mathbb{R P}^{n}$ is the canonical line bundle over $\mathbb{R P}^{n}$, is strongly algebraic because its StiefelWhitney class $w_{1}\left(\tilde{f}^{*}\left(\gamma_{n, 1}\right)\right)=v$ is in $H_{\text {alg }}^{1}\left(X, \mathbb{Z}_{2}\right)$ (cf. Theorem 12.4.5 [2]). Now by Theorem 13.3.1 of [2], the map $\tilde{f}$ classifying the pull-back bundle can be homotoped to an entire rational map.

\section{REFERENCES}

1. S. Akbulut, H. King, Topology of Real Algebraic Sets, M.S.R.I. book series (Springer, New York, 1992). MR1225577 (94m:57001)

2. J. Bochnak, M. Coste, M.F. Roy, Real Algebraic Geometry, Ergebnisse der Math., vol. 36 (Springer, Berlin, 1998). MR.1659509 (2000a:14067)

3. J. Bochnak and W. Kucharz, On real algebraic morphisms into even-dimensional spheres, Ann. of Math. (2), 128 (1988) 415-433. MR960952 (89k:57060)

4. J. Bochnak, M. Buchner and W. Kucharz, Vector bundles over real algebraic varieties, K-Theory, 3 (1990) 271-298. MR 1040403 (91b:14075)

5. J. Bochnak and W. Kucharz, Algebraic approximation of mappings into spheres, Michigan Math. J., 34 (1987) 119-125. MR873026 (88h:58018)

6. J. Bochnak and W. Kucharz, Complex cycles on real algebraic models of a smooth manifold, Proc. Amer. Math. Soc., 114 (1992) 1097-1104. MR1093594 (93g:57032)

7. J. Bochnak and W. Kucharz, Algebraic models of smooth manifolds, Invent. Math., 97 (1989) 585-611. MR 1005007 (91b:14076)

8. G.E. Bredon, Topology and Geometry (Springer, New York, 1993). MR1224675 (94d:55001)

9. N. Ivanov, Approximation of smooth manifolds by real algebraic sets, Russian Math. Surveys, 37 (1982) 1-59. MR643764(84i:57029)

10. J. L. Loday, Applications algébriques du tore dans la sphère et de $S^{p} \times S^{q}$ dans $S^{p+q}$, Lect. Notes in Math 342 (Springer, 1973). MR0368034(51:4276)

11. Y. Ozan, On entire rational maps in real algebraic geometry, Michigan Math. J., 42 (1995) 141-145. MR1322195 (96b:14070)

12. Y. Ozan, On homology of real algebraic varieties, Proc. Amer. Math. Soc., 129 (2001) 31673175. MR.1844989 (2002m:14048)

Department of Mathematics, Abant İzzet Baysal University, 14280 Bolu, Turkey

E-mail address: ozturkali@ibu.edu.tr 